\title{
The Types and Pattern of Use of Mobile Health Applications Among the General Population: A Cross-Sectional Study from Selangor, Malaysia
}

\author{
Bhuvan KC (iD) ${ }^{1,2}$ \\ Alian A Alrasheedy (iD ${ }^{3}$ \\ Bey Hing Goh (iD) 4,5 \\ Ali Blebil (iD) \\ Nida Sajid Ali Bangash iD ' \\ Mohamed Izham Mohamed \\ Ibrahim iD ${ }^{6}$ \\ Inayat Ur Rehman ${ }^{7}$ \\ 'School of Pharmacy, Monash University \\ Malaysia, Selangor, 47500, Malaysia; \\ ${ }^{2}$ College of Public Health, Medical and \\ Veterinary Sciences, James Cook \\ University, Townsville, Australia; \\ ${ }^{3}$ Department of Pharmacy Practice, \\ College of Pharmacy, Qassim University, \\ Buraidah, Qassim, Saudi Arabia; \\ ${ }^{4}$ Biofunctional Molecule Exploratory \\ (BMEX) Research Group, School of \\ Pharmacy, Monash University Malaysia, \\ Bandar Sunway, 47500, Malaysia; ${ }^{5}$ College \\ of Pharmaceutical Sciences, Zhejiang \\ University, Hangzhou, 310058, People's \\ Republic of China; ${ }^{6}$ College of Pharmacy, \\ QU Health, Qatar University, Doha, \\ Qatar; ${ }^{7}$ Department of Pharmacy, Abdul \\ Wali Khan University Mardan, Mardan, \\ Pakistan
}

Purpose: The aims of this study are to explore the types and patterns of use of health mobile apps among pharmacy clients in Selangor, Malaysia, and to explore the users' experiences and views regarding the benefits of and issues with the use of the health apps.

Patients and Methods: A cross-sectional survey was conducted with 278 pharmacy clients who were using health apps on their smartphones. A survey was developed, piloted, and rolled out, comprised of socio-demographic characteristics, information regarding the common types and the pattern of health app use, the benefits of health apps, and issues that users faced while using these apps. Statistical analysis was performed using IBM SPSS version 21.

Results: The mean $( \pm \mathrm{SD})$ age of health app users was $29.8( \pm 11.74)$ years old, and over half of them $(51.8 \%)$ were male, while less than half were female $(48.2 \%)$. There were 48 different types of mobile health apps used by the participants in this study. The two most common were multi-purpose general health apps (53.6\%) and fitness apps (38.1\%). In terms of pattern of use, $33.5 \%$ of the participants reported using their health apps daily, $36.7 \%$ at least once weekly, and $25.2 \%$ monthly. The main benefits reported by the users were tracking of health status (47\%), motivation (41\%), and knowledge about health and fitness (9\%). The main issues reported by the participants included inaccuracy of the app (24\%), inconvenience (20.7\%), and not being user-friendly (18.5\%).

Conclusion: The study showed that the participants used many different types of mobile health apps for several purposes, including general health, wellness, fitness, and selfmanagement of diseases. These apps helped the participants to track their health-related activities and motivated them to maintain their wellness and fitness. Further areas of improvement were identified to ensure evidence-based and effective utilization of apps to achieve required health outcomes.

Keywords: digital health, digital patients, health apps, Malaysia, mobile health

\section{Introduction}

The advancement of, and improved access to, information and communications technology (ICT) applications has led to a tremendous increase in the use of smartphones among Malaysians. ${ }^{1-3}$ A smartphone is "a mobile phone that has a touchscreen interface, internet accessibility, is capable of downloading apps and possesses many functions of a computer". In Malaysia, the 2020 internet-user survey showed that Malaysians are widely using ICT and the internet in their daily activities, and that the top five online activities involve using text communication (98.1\%), using social media (93.3\%), watching videos (87.3\%), using voice/video
Correspondence: Bhuvan KC School of Pharmacy, Monash University Malaysia, Jalan Lagoon Selatan, Bandar

Sunway, Selangor, 47500, Malaysia

$\mathrm{Tel}+60142271852$

Email bhuvan.kc@monash.edu 
communication (81.1\%), and getting information $(74.3 \%) .{ }^{1}$ The survey also showed that the number of internet users and different internet-based activities increased in 2020 compared to 2018. Moreover, the 2020 survey reported that the use of smartphones to access the internet has almost reached a saturation point at $98.7 \%$ of users. ${ }^{1}$ This surge in the use of smartphones with greater access to the internet provides suitable grounds for the greater use of mobile health applications (apps) among urban dwellers in Malaysian cities.

In the health care sector, the use of mobile health apps is a relatively new phenomenon; however, their use for both general health and specific medical purposes is increasing globally. ${ }^{5}$ Krebs and Duncan conducted a study with 1604 mobile phone users throughout the United States of America (USA), and 58.23\% reported that they had downloaded mobile health apps and were using them mostly for fitness and nutrition. ${ }^{6}$ In another study among primary care patients in California, USA, Ramirez et al reported that $31.50 \%$ of mobile phone users with mobile apps on their phones used health apps, and the majority $(86 \%)$ reported an interest in using mobile apps to improve their health. ${ }^{7}$

For specific diseases, a comprehensive review concluded that mobile health apps are beneficial and have the potential to improve the management of Alzheimer's disease and related dementias by providing useful caregiving information. However, the review identified several areas for improvement, to maximize the health benefits of the use of health apps for patients with Alzheimer's disease and related dementias (eg, improvement of content and format of the apps, such as having more audio/video options). ${ }^{8}$

The use of mobile health apps has several challenges. A study by Boyle et al in New Zealand among diabetesrelated mobile health app users and the healthcare providers treating them revealed that many healthcare providers did not feel confident with the apps' insulin dose calculation feature. ${ }^{9}$ The study suggested the need for a proper assessment of mobile health apps to improve their quality and safety features for both application users and healthcare providers. ${ }^{9}$ Moreover, in a systematic review, Santo et al reported that many medication-reminder mobile apps are available on the market, but the majority did not have the desirable features and were of low quality. ${ }^{10}$

In Malaysia, with a greater internet penetration and smartphones becoming more accessible to the general public, the use of mobile health apps is currently increasing. In a study carried out among healthcare providers who used mobile health apps for adverse drug reaction (ADR) assessment, Ithnin et al found that the use of such apps could positively contribute to the detection and assessment of ADRs and to ensuring medication safety. ${ }^{11}$ However, in a recent study in Malaysia that included 4504 participants, Lee et al reported that the majority of participants were not familiar with the term "mobile health" (m-Health) but, upon hearing about it, were positive towards its use. ${ }^{12}$ The study also highlighted that people were willing to use mobile health apps to better manage their health conditions but wanted more education and training. ${ }^{12}$ Consequently, there is a need to explore in more detail the types, pattern of use, functions, perceived benefits, and issues/challenges with mobile health apps among Malaysian smartphone users as there is a dearth of information in this regard.

Both individuals and the healthcare system can benefit from mobile health apps only when the following crucial information is available: a) the availability and usability features of mobile health apps that are available and used in Malaysia, b) the types of mobile health apps used by consumers and for what purpose, and c) users' perception and experience with different mobile health apps. However, such studies are currently lacking, although a study by Hussein et al was conducted in Sarawak, Malaysia to explore the characteristics of smartphone users, associated with the intentions to use mobile health apps. The authors reported that the participants' intentions to use the health apps were not associated with their characteristics (ie, gender, age, level of education, and health condition). Consequently, they suggested that developers of mobile health apps need to promote these apps to patients and consumers to increase awareness and usability. ${ }^{13}$ Chew et al reported encouraging findings on a newly developed medication adherence app in Malaysia and showed that it had the potential to improve medication adherence among ambulatory care patients in Malaysia. ${ }^{14}$ These studies reinforce the need to carry out further studies among the general population that focus on the current types and pattern of use, and on users' views regarding mobile health apps in Malaysia. Thus, the aims of this study are to explore the types and pattern of use of health apps among pharmacy clients in the Selangor State of Malaysia, and to explore their experiences and views regarding the benefits of, and issues with, the use of health apps. Consequently, based on the findings, implications and recommendations are made to provide further guidance on this topic to health policymakers and healthcare professionals in order to promote the safe and evidence-based use of health apps. 


\section{Patients and Methods}

\section{Study Design, Setting and Population}

A cross-sectional descriptive study was conducted with the general Malaysian population who were using mobile health apps in the state of Selangor, Malaysia. The participants in the study were recruited from retail pharmacies and health stores located in different shopping malls in the cities of Kuala Lumpur and Subang Jaya, State of Selangor, Malaysia. Clients visiting the pharmacies and health stores were approached and invited to participate in the study, and those who agreed were interviewed. The inclusion criteria included smartphone owners who were using health-related apps, of age $\geq 18$ years old, and those who could speak the English language.

\section{The Questionnaire Development}

A literature review on different aspects of mobile health apps was carried out, including reports and documents about ICT and mobile health app use in Malaysia. Following this, a questionnaire was developed. The draft questionnaire was then given to a review team comprising public health and ICT experts for face and content validation, before being piloted with 15 individuals who were using mobile health apps. Comments, suggestions and feedback during the pilot testing were incorporated, and subsequent minor modifications were made. The final questionnaire comprised questions/items related to sociodemographics (ie, gender, age, ethnicity, and education) and information related to health apps (ie, common types of apps, pattern of use, the benefits of using health apps, and the issues/challenges faced while using these apps).

\section{Data Collection Strategy}

This was an interviewer-administered questionnaire. Smartphone owners visiting pharmacies and other health stores were approached, and those who were using mobile health apps and met the inclusion criteria were invited to participate in the study. The participants were briefed on the objective of the study, and a written consent was taken before participation in the study. The study was conducted during the period from January 2019 to March 2019.

\section{Data Analysis}

Data analysis was performed using IBM SPSS for Windows, Version 21.0. Armonk, NY: IBM Corp. Descriptive analysis was used to analyze the data. The categorical variables were summarized using frequency and percentage, and the continuous variables were summarized using the mean and standard deviation (SD), and the range.

\section{Ethical Approval}

Ethics approval was obtained from the Monash University Human Research Ethics Committee (Project ID: 18215, date of approval: 14/01/2019) prior to study commencement. The study was conducted in accordance with the Declaration of Helsinki.

\section{Results}

\section{Socio-Demographic Data}

A total of 278 participants took part in the study. As shown in Table $1,51.8 \%$ of the sample were male and $48.2 \%$ were female, and the mean age (SD) of participants was 29.76 (11.74). Furthermore, in terms of ethnicity, 69\% of the participants were Chinese. The participants were from different educational backgrounds (Table 1).

\section{Types of Health Apps Used by the Participants}

Different types of mobile health apps are available via the Apple App Store or the Google Play Store. In this

Table I Socio-Demographic Characteristics $(n=278)$

\begin{tabular}{|c|c|c|}
\hline Variable & Number & Percentage \\
\hline \multicolumn{3}{|l|}{ Age } \\
\hline Mean \pm SD & \multirow{2}{*}{\multicolumn{2}{|c|}{$\begin{array}{l}29.76 \pm 11.74 \\
18-65\end{array}$}} \\
\hline Range & & \\
\hline \multicolumn{3}{|l|}{ Gender } \\
\hline Male & 144 & 51.8 \\
\hline Female & 134 & 48.2 \\
\hline \multicolumn{3}{|l|}{ Ethnicity } \\
\hline Malay & 52 & 18.7 \\
\hline Chinese & 193 & 69.4 \\
\hline Indian & 31 & 11.2 \\
\hline Others & 2 & 0.7 \\
\hline \multicolumn{3}{|l|}{ Education } \\
\hline Pre-university level & 34 & 12.2 \\
\hline Diploma & 88 & 31.7 \\
\hline Bachelor's degree & 81 & 29.1 \\
\hline Master's degree & 13 & 4.7 \\
\hline Professional degree & 11 & 4.0 \\
\hline NA & 51 & 18.3 \\
\hline
\end{tabular}

Abbreviation: NA, not available. 


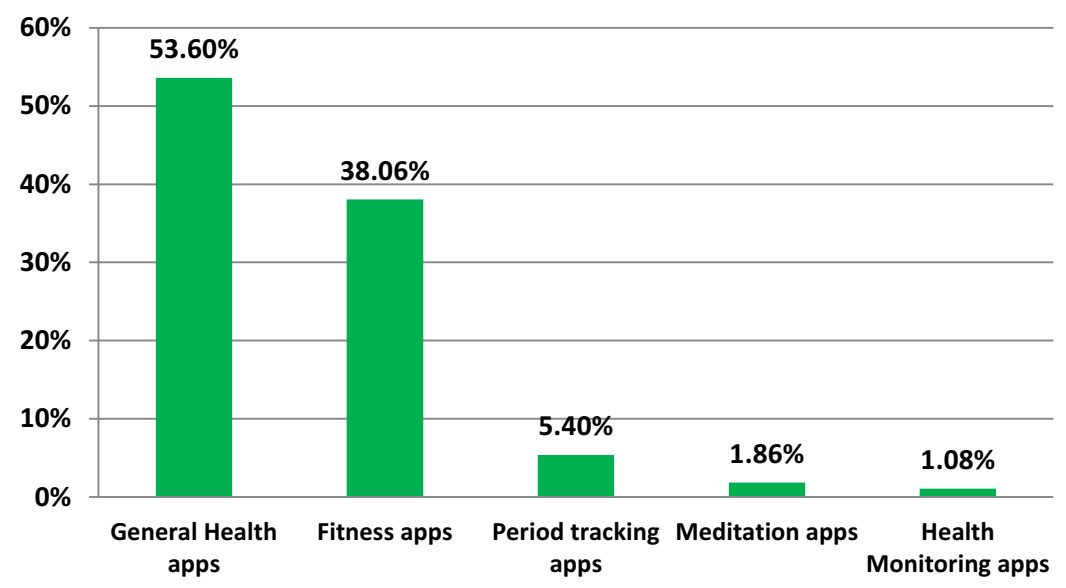

Figure I Common types of mobile health apps used by the participants.

study, a total of 48 different health related apps were used by the 278 participants. As shown in Figure 1 and in Supplementary Table 1, the participants used different types of mobile health apps for various purposes, and the types of apps included multi-purpose health apps, fitness apps, period tracking apps, meditation apps, and health monitoring apps.

\section{The Pattern of Use of the Health Apps}

Table 2 shows the participants' usage frequency of mobile health apps. In this study, $33.5 \%$ of the participants reported using their health apps on a daily basis, $36.7 \%$ at least once weekly, and $25.2 \%$ monthly.

\section{The Participants' Views Regarding the} Benefits and Challenges with the Use of Mobile Health Apps

The main benefits of mobile health apps were tracking of health status (47\%), followed by motivation (41\%) and gaining knowledge about health and fitness $(9 \%)$. The

Table 2 Usage Frequency of Mobile Health App

\begin{tabular}{|l|l|l|}
\hline Pattern & Frequency & Percentage* \\
\hline Daily & 93 & 33.5 \\
Weekly & 102 & 36.7 \\
Monthly & 70 & 25.2 \\
Twice a week & 6 & 2.2 \\
Twice a month & 1 & 0.4 \\
NA & 6 & 2.2 \\
Total & 278 & 100.0 \\
\hline
\end{tabular}

Note: *The percentage does not strictly add up to $100 \%$ due to rounding. Abbreviation: NA, not available. main issues reported by the participants included inaccuracy of the app (24\%), inconvenience (20.7\%), and not being user-friendly (18.5\%).

\section{Participants' Sharing of Their Health Data Generated by Their Apps}

As shown in Table 3, most participants (83.5\%) reported that they did not share their data and information generated by their mobile health apps with others, including their healthcare professionals. Only a few participants (8.3\%) reported sharing their health data from these apps with their medical doctors or pharmacists.

\section{Use of Health Apps for Self-Management of Chronic Diseases}

Most of the users of the health apps were healthy young adults and had no chronic conditions. However, some participants with chronic diseases $(n=35)$ reported using mobile health apps for managing disease conditions, such as diabetes and hypertension.

\section{Discussion}

The findings from the current study showed that the participants used a variety of mobile health apps for various health and wellness purposes. The diversity of users and mobile health apps among the public also highlights the scope for integration of mobile health apps with the Malaysian health system. Furthermore, the findings from the current study in Malaysia are in line with other studies from the USA and Denmark, which showed that young adults were more likely to use mobile health apps as they 
Table 3 Participants' Sharing Outcomes of Mobile Health Apps with Their Healthcare Professionals and Others

\begin{tabular}{|l|l|l|}
\hline Variable & Frequency & Percentage* \\
\hline Did not share/personal use only & 232 & 83.5 \\
Medical doctor & 20 & 7.2 \\
Pharmacist & 3 & 1.1 \\
Family & 7 & 2.5 \\
Friends & 8 & 2.9 \\
Family and Friends & 6 & 2.2 \\
Social group & 2 & 0.7 \\
Total & 278 & 100.0 \\
\hline
\end{tabular}

Note: *The percentage does not strictly add up to $100 \%$ due to rounding.

are more technology savvy (ie, have higher levels of e-health literacy skills). ${ }^{6,15}$ The increasing use of mobile health apps provides a foundation for the use of these apps by the health systems. ${ }^{6,16}$ Nevertheless, this needs a comprehensive approach in terms of the standardization of mobile health apps, educating patients/target groups, and creating a mechanism to use data from mobile health apps within the health system.

In Malaysia, the 2020 internet survey indicated a very high usage of the internet, estimated at $88.7 \%$ of the population. In addition, approximately $71 \%$ used the internet for $\geq 5$ hours daily. Moreover, the survey reported that accessing the internet through smartphones almost reached saturation level at $98.7 \%$ in $2020 .{ }^{1}$ Furthermore, the hand phone users survey (HPUS) conducted in Malaysia showed that the percentage of smartphone users increased to almost $80 \%$ in 2018 with a high adoption of smartphones, including among the low socioeconomic population. ${ }^{3}$ These facts provide opportunities for the healthcare system to increase access to quality healthcare through mobile health and to expand digital health services, including teleconsultation. Graetz et al reported that mobile health-accessible personal health records among diabetic patients can help bridge the digital divide due to a lack of computer access among those of lower socioeconomic status. ${ }^{17}$ The penetration of mobile health apps among people of differing socioeconomic statuses and demographics is a good thing as it helps to improve access to health services in Malaysia and in other countries as well.

Most of the study participants (53.6\%) had downloaded multi-purpose health apps, including the pre-installed health apps provided by the smartphone manufacturers. In addition, among the health-related apps, fitness-related apps were most frequently used by the participants $(38.06 \%)$, with the majority using health-related apps on a daily or weekly basis. Krebs and Duncan also reported that a similar proportion (58.2\%) of mobile phone users had downloaded health-related mobile apps that were fitness and nutrition related, with most respondents using them at least daily. ${ }^{6}$ Reasons for using these healthrelated mobile apps included supporting health-related behavior changes, tracking progress in diet and exercise, getting feedback such as an estimated weight loss or gain, and finding out how far they were from their goal weight. Built-in mobile health apps can be a way to increase the use of mobile health apps for other diseases and health purpose, but more studies are needed to investigate these aspects.

The participants in our study cited three main reasons to use and download the health apps on their smartphones, namely health status tracking, motivation, and knowledge about their health and fitness. Therefore, the features and specifications of health apps that are required to satisfy patients' needs and preferences are considered to be important aspects to expand the use of mobile health apps. Mendiola et al reported that mobile apps that are simple, easy to use, come with instructions to manage a condition, and allow them to share data with designated individuals are deemed to be valuable by users. ${ }^{18}$ Specific details, usage guidelines, data, other shareable features, and strong motivation might be the reasons that mobile health apps for exercise and fitness are successful and commonly used. ${ }^{19,20}$ However, only a very few of the popular exercise- and fitness-related mobile health apps were evidence-based and followed various health/clinical guidelines. ${ }^{21}$ In a study on system design and critical issues of mobile health apps, Baig et al reported that issues and challenges related to security and privacy of data, acceptability, reliability, and cost need to be addressed in order to benefit from mobile apps for patient monitoring, education, etc. ${ }^{22}$ Consequently, comprehensive and concerted efforts are needed to ensure the effective, evidencebased and safe use of mobile apps for healthcare and other medical purposes for a long period of time. ${ }^{22-24}$ These needs provide an opportunity for health app developers to provide quality and evidence-based health apps with user friendly features. ${ }^{21}$

Our study also shows that some of the participants used disease-specific mobile health apps. However, only a small proportion of people were using mobile health apps related to healthcare problems such as hypertension, diabetes, etc. 
This was expected, as most of the health app users were relatively young and healthy. These findings are similar to the results from a national survey in the USA. ${ }^{6}$ The use of mobile health apps can help patients better manage their chronic health problems; however, such a result would require the use of mobile health apps on a continued basis and in accordance with the plan of a medical practitioner for better therapeutic outcomes. This is because using health apps for a short-term period or stopping them after initial adoption (ie, after a few times of use) limits the user from achieving the intended outcomes and any potential behavioral changes. ${ }^{25}$ For example, Cedars and Blackmore reported that a mobile health app for congenital heart diseases was potentially helpful in improving the quality of care and also facilitating communication between patient and providers, but patient compliance in terms of regular use was poor. ${ }^{26}$ Overall, it is believed that mobile health apps have the potential to be used for better management of chronic health conditions, but improvements are required in terms of design, usability features, ease of use, and careful integration into a patient selfmanagement plan by healthcare professionals. Future studies need to focus on the use of specific mobile health apps, tailored for specific health conditions, that are integrated into a patient management plan. ${ }^{27,28}$

The study has several implications. There is a great opportunity for digital health apps in Malaysia, particularly mobile health apps, given the wide use of smartphones and the wide internet coverage throughout the country. Therefore, health apps have the potential to promote healthy lifestyles and to empower patients in their self-management. However, we believe more efforts are needed from pertinent stakeholders in this regard to ensure the safe and effective utilization of health apps, with appropriate measures to maintain security and confidentiality of patients' data. First, the app developers could work with relevant professional health societies to ensure that the apps are built based on clinical guidelines and the best available evidence. Moreover, app developers need to constantly review the feedback from their users to improve their apps' design, features, etc. In addition, on a global level, since apps can be downloaded and used by different clients and patients anywhere in the world (ie, digital consumers), health authorities could develop a system for certifying health apps, taking clinical and technical aspects into consideration to ensure their quality. Based on this, healthcare professionals could also promote the evidence-based use of health apps to their patients to effectively improve adherence to a plan and management of their health, including motivation to maintain healthy lifestyles. In the near future, the use of mobile health apps and their types/purposes are expected to increase at an unprecedented rate. Pharmacists should be well prepared to guide patients and to empower them to be healthier and to manage their diseases better as they use the different types of available apps.

\section{Strengths and Limitations of the Study}

This is one of the few studies that has explored consumers' perspectives towards mobile health apps in Malaysia. Moreover, the study explored several aspects related to the use of health apps. However, it has some limitations. The study was conducted in only one state; therefore, the findings might not be generalizable to other states. In addition, there were only a few consumers using diseasespecific apps (eg, apps for hypertension or diabetes management); hence, we recommend that future studies explore the usage of disease-specific apps in pertinent patient populations (eg, patients with diabetes). Moreover, apps that were not in the English language (eg, Mandarin, Malay, Tamil) were excluded from this study; thus, mobile health apps covered in this study may not be representative of all apps available in the state of Selangor. However, overall, we believe that the current study's findings can be useful and can help to provide further guidance to health policymakers, digital health companies and healthcare professionals on the use of digital health in the healthcare system.

\section{Conclusion}

The study showed that the participants used many different types of mobile health apps. In addition, they used these health apps for several purposes, including general health, wellness, fitness, and, for some, the self-management of diseases. These apps helped the participants to track and better manage their health and motivated them to maintain their wellness and fitness. However, further areas of improvement were identified to ensure evidence-based and effective utilization of apps to achieve the required health outcomes.

\section{Abbreviations}

ADRs, adverse drug reactions; Apps, applications; HPUS, hand phone users survey; ICT, information and 
communications technology; m-Health, mobile health; SD, standard deviation; USA, United States of America.

\section{Acknowledgments}

We would like to acknowledge the efforts of Mr Sunil Shrestha and Ms Asmita Priyadarshini Khatiwada for their help with different aspects of data analysis and manuscript formatting.

\section{Funding}

This research did not receive any specific grant from funding agencies in the public, commercial, or not-forprofit sectors.

\section{Disclosure}

The authors report no conflicts of interest in this work.

\section{References}

1. Malaysian Communications and Multimedia Commission. Commission Internet User Survey 2020; Updated June 2021. Available from: https://www.mcmc.gov.my/skmmgovmy/media/ General/pdf/IUS-2020-Report.pdf. Accessed June 2, 2020.

2. Osman MA, Talib AZ, Sanusi ZA, Shiang-Yen T, Alwi AS. A study of the trend of smartphone and its usage behavior in Malaysia Int J New Comput Archit Appl. 2012;2(1):274-285.

3. Malaysian Communications and Multimedia Commission. Commission Hand Phone Users Survey 2018; Updated June 2021. Available from: https://www.mcmc.gov.my/skmmgovmy/media/ General/pdf/HPUS2018.pdf. Accessed June 1, 2020.

4. Lexico.com. Smartphone [Internet]. Oxford: Oxford University Press; 2019; Updated 2021. Available from: https://en.oxforddiction aries.com/definition/smartphone. Accessed April 2, 2021.

5. Qiang C, Yamamichi M, Hausman V, Miller R, Altman D Mobile applications for the health sector. World Bank; 2012 [cited April 2, 2021]. Available from: https://documents1.worldbank.org/curated/en/ 751411468157784302/pdf/726040WP0Box370th0report00Apr020120. pdf. Accessed August 3, 2021.

6. Krebs P, Duncan DT. Health app use among US mobile phone owners: a national survey. JMIR mHealth uHealth. 2015;3(4):e101. doi: 10.2196/mhealth.4924

7. Ramirez V, Johnson E, Gonzalez C, Ramirez V, Rubino B, Rossetti G. Assessing the use of mobile health technology by patients: an observational study in primary care clinics. JMIR mHealth uHealth. 2016;4(2):e41. doi:10.2196/mhealth.4928

8. Ezeanya V, Choi SK, Friedman D, Kannaley K. Review of alzheimer's disease focused mobile applications. Iproceedings. 2017;3(1) e44. doi:10.2196/iproc. 8460

9. Boyle L, Grainger R, Hall RM, Krebs JD. Use of and beliefs about mobile phone apps for diabetes self-management: surveys of people in a hospital diabetes clinic and diabetes health professionals in New Zealand. JMIR mHealth uHealth. 2017;5(6):e85. doi:10.2196/ mhealth. 7263

10. Santo K, Richtering SS, Chalmers J, Thiagalingam A, Chow CK, Redfern J. Mobile phone apps to improve medication adherence: a systematic stepwise process to identify high-quality apps. JMIR mHealth uHealth. 2016;4(4):e132. doi:10.2196/mhealth.6742
11. Ithnin M, Rani MDM, Abd Latif Z, et al. Mobile app design, development, and publication for adverse drug reaction assessments of causality, severity, and preventability. JMIR mHealth uHealth. 2017;5(5):e78. doi:10.2196/mhealth.6261

12. Lee JY, Wong CP, Lee SWH. m-Health views and perception among Malaysian: findings from a survey among individuals living in Selangor. Mhealth. 2020;6:6. PubMed PMID: 32190617. eng. doi:10.21037/mhealth.2019.09.16

13. Hussein Z, Harun A, Oon SW. The influence of the smartphone user's characteristics on the intention to use of M-health. Int J Humanit Soc Sci. 2016;2(5):598-602.

14. Chew S, Lai PS, Ng CJ. Usability and utility of a mobile app to improve medication adherence among ambulatory care patients in Malaysia: qualitative study. JMIR mHealth uHealth. 2020;8(1): e15146. doi:10.2196/15146

15. Bol N, Helberger N, Weert JCM. Differences in mobile health app use: a source of new digital inequalities? Inf Soc. 2018;34 (3):183-193. doi:10.1080/01972243.2018.1438550

16. Jusoh S. A survey on trend, opportunities and challenges of mHealth apps. Int J Interact Mob Technol. 2017;11(6):73-85. doi:10.3991/ ijim.v11i6.7265

17. Graetz I, Huang J, Brand R, Hsu J, Yamin CK, Reed ME. Bridging the digital divide: mobile access to personal health records among patients with diabetes. Am J Manag Care. 2018;24(1):43.

18. Mendiola MF, Kalnicki M, Lindenauer S. Valuable features in mobile health apps for patients and consumers: content analysis of apps and user ratings. JMIR mHealth uHealth. 2015;3(2):e40. doi:10.2196/ mhealth. 4283

19. Rabin C, Bock B. Desired features of smartphone applications promoting physical activity. Telemed J E Health. 2011;17(10):801-803. doi: $10.1089 / \mathrm{tmj} .2011 .0055$

20. West JH, Belvedere LM, Andreasen R, Frandsen C, Hall PC, Crookston BT. Controlling your "app" etite: how diet and nutritionrelated mobile apps lead to behavior change. JMIR mHealth uHealth. 2017;5(7):e95. doi:10.2196/mhealth.7410

21. Modave F, Bian J, Leavitt T, Bromwell J, Harris Iii C, Vincent H. Low quality of free coaching apps with respect to the American College of Sports Medicine Guidelines: a review of current mobile apps. JMIR mHealth uHealth. 2015;3(3):e77. doi:10.2196/mhealth.4669

22. Baig MM, GholamHosseini H, Connolly MJ. Mobile healthcare applications: system design review, critical issues and challenges. Phys Eng Sci Med. 2015;38(1):23-38. doi:10.1007/s13246-0140315-4

23. Dennison L, Morrison L, Conway G, Yardley L. Opportunities and challenges for smartphone applications in supporting health behavior change: qualitative study. J Med Internet Res. 2013;15(4):e86. doi:10.2196/jmir.2583

24. Angosto S, García-Fernández J, Valantine I, Grimaldi-Puyana M. The intention to use fitness and physical activity apps: a systematic review. Sustainability. 2020;12(16):6641. doi:10.3390/su12166641

25. Vaghefi I, Tulu B. The continued use of mobile health apps: insights from a longitudinal study. JMIR mHealth uHealth. 2019;7(8):e12983. doi: $10.2196 / 12983$

26. Cedars A, Blackmore C, editors. Use of a Disease-Specific Mobile Health Application in the Care of Adults with Congenital Heart Disease. Baylor University Medical Center Proceedings. Taylor \& Francis; 2019.

27. Apolinário-Hagen J, Menzel $M$, Hennemann S, Salewski C. Acceptance of mobile health apps for disease management among people with multiple sclerosis: web-based survey study. JMIR Form Res. 2018;2(2):e11977. doi:10.2196/11977

28. Mano R. Use of mobile health applications and the self-management of cancer: a gendered approach. Open J Prev Med. 2019;9(10):126. doi:10.4236/ojpm.2019.910012 


\section{Publish your work in this journal}

Patient Preference and Adherence is an international, peer-reviewed, open access journal that focusing on the growing importance of patient preference and adherence throughout the therapeutic continuum. Patient satisfaction, acceptability, quality of life, compliance, persistence and their role in developing new therapeutic modalities and compounds to optimize clinical outcomes for existing disease states are major areas of interest for the journal. This journal has been accepted for indexing on PubMed Central. The manuscript management system is completely online and includes a very quick and fair peer-review system, which is all easy to use. Visit http:// www.dovepress.com/testimonials.php to read real quotes from published authors. 